This Technical Report is also published as Chapter 19 IN

M-Health: Emerging Mobile Health Systems, Robert H. Istepanian, Swamy Laxminarayan, Constantinos S. Pattichis, Editors, 2006.

Springer. $624 \mathrm{p}$.

ISBN: 0-387-26558-9.

AS

FUTURE CHALLENGES AND RECOMMENDATIONS

Val Jones, F. Incardona, C. Tristram, S. Virtuoso, A. Lymberis 


\title{
FUTURE CHALLENGES AND RECOMMENDATIONS
}

\author{
Val Jones, F. Incardona, C. Tristram, S. Virtuoso, A. Lymberis
}

Rapid advances in information technology and telecommunications, and in particular mobile and wireless communications, converge towards the emergence of a new type of "infostructure" that has the potential of supporting a large spectrum of advanced services for healthcare and health. Currently the ICT community produces a great effort to drill down from the vision and the promises of wireless and mobile technologies and provide practical application solutions. Research and development include data gathering and omni-directional transfer of vital information, integration of human machine interface technology into handheld devices and personal applications, security and interoperability of date and integration with hospital legacy systems and electronic patient record. The ongoing evolution of wireless technology and mobile device capabilities is changing the way healthcare providers interact with information technologies. The growth and acceptance of mobile information technology at the point of care, coupled with the promise and convenience of data on demand, creates opportunities for enhanced patient care and safety. The developments presented in this section demonstrate clearly the innovation aspects and trends towards user oriented applications.

However, several major challenges still need to be addressed in order to broaden the implementation and use of mobile health devices and services and strengthen the market development.

The main obstacles to the penetration of mobile solutions based on handheld computerised devices and wireless technology in the healthcare sector can be roughly classified into objective and subjective:

Objective obstacles have to do with deficiencies and limitations of the current technology, specifically interoperability, compliance to widely accepted standards and security. Interoperability and standards issues are especially relevant, as they often impede or make a tough job to integrate mobile solutions into the framework of existing systems currently in healthcare institutions for information storage and management purposes. To help overcome this difficulty, the adoption of open standards and cross-platform technology is advised wherever possible. Where this is not possible it is recommended that a layer be constructed on the legacy system with a standards compliant interface to the external world. Access control and security are crucial to the eventual acceptance of mobile solutions by the healthcare community and should not be underestimated. The confidential nature of the data to be accessed obviously requires that access rights be carefully matched against user authentication parameters. Wired network solutions usually approach the problem by adopting security models based on digital certificates and electronic signatures. This may not feasible with the mobile devices currently available on the market. On the contrary, the first results obtained in this respect by some of the projects presented in this section (e.g. Mobi-Dev) show a substantial immaturity of PKI technology for mobile platforms - problematic support for smart card readers, unreliable functioning of available cryptoAPI libraries for digital certificate management, pocket browsers not enabling client authentication in SSL sessions etc. These problems may be overcome when new PDA models and OS versions become available. It remains however a strong recommendation that transmissions be encrypted, authentication be included in non-revocable transactions from the mobile devices to the legacy and new systems and that the data be stored in an encrypted format with access being dependent on certificated keys

Subjective obstacles concern the reluctance of healthcare professionals - often busy people with techno-phobic attitudes and little time left for technical training - to abandon traditional mostly paper and voice based workflows for new practices based on highly technological appliances such as new generation PDAs. This is especially true of applications that require excessively awkward or lengthy procedures. The experience accumulated so far evidences the importance of parameters such as 
user-friendliness, lightness and functionality in application design, even at the cost of realizing less attractive user interfaces.

A further major obstacle to the adoption of advanced mobile solutions in the healthcare sector is related to the need to comply with usually very restrictive regulations. These are designed to ensure decision traceability in each step of care delivery and guarantee the patients against privacy violations. This is a quite delicate issue that can often only be dealt with by promoting actions aimed at awakening in the legislators the necessary appreciation for the benefits deriving to the healthcare system from the adoption of information technology mature solutions.

Several technical aspects remain to be solved, starting with existing constraints e.g. screen size, resolution and battery life.

Vocal input is a major goal in order to increase usability of mobile devices and applications. It is presently approached in two ways: single word discrete voice recognition, where speech recognition is carried on selecting from long lists; and continuous speech recognition with natural language understanding. This second approach must be included in an architecture in which dictation is entered into the MMD and the recognition is done on a server (this architecture has been adopted in the Mobi-Dev project). Although this is a clear limitation, so as the low quality of the incorporated microphones, there remain considerable opportunities for improved user interfaces enabling vocal input. This is being investigated by the MEMO project as an implementation for SMARTIE and MobiDev an associated project.

Security remains a major issue for authentication of the user as well as encryption of transmitted data. Within the associated to MEMO projects the use of smart cards has been investigated, where the smart card holds encryption codes and authentication certificates. Contacts have been made with other suppliers to identify the feasibility of adding biometric based smart cards to MMDs.

Initial investigations into remote communications architectures and technologies indicate that for many cases GPRS will be an adequate substitute for UMTS based devices for the foreseeable future. However, for other applications we are already beyond the capabilities of UMTS (eg 12-lead ECG transmission or EEG, not to mention VR and AR applications and rendering 3D graphics. Local communications are shown to work well with both wireless LAN and Bluetooth. The difference in usage is dependent upon distance and numbers of devices connected. The main constraint in this area at the present time is the cost of Bluetooth devices and the power of Wireless LAN which is being addressed by the widespread introduction of WiFi systems.

Integration of mobile platforms with existing systems can be pursued by adopting the approaches usually followed in ordinary Enterprise Application Integration (EAI). The integration between different information systems, possibly legacy systems using obsolescent technology, is normally realised by interposing in between a middleware software layer, responsible of mediating between two separate and already existing software platforms, typically DBMSs. EAI may involve developing a new total view of an organisation's business and its applications, seeing how existing applications fit into the new view, and then devising ways to efficiently reuse what already exists while adding new applications and data. This is made possible by using methodologies such as object-oriented programming, distributed, cross-platform program communication using message brokers with Common Object Request Broker Architecture and $\mathrm{COM}+$, organisation-wide content and data distribution using common databases and data standards implemented with the Extensible Markup Language (XML) (both data and voice $\mathrm{XML}$ ), middleware, message queuing, intelligent agents and other approaches. The common belief that application integration is a short-term patch to apply while awaiting a perfectly interoperable, homogenous application base is a myth. Heterogeneity is an intrinsic characteristic of information systems, and which can hardly be dismissed. Networkoriented development platforms such as Java 2 Enterprise Edition (J2EE) or Microsoft's .NET are not going to solve semantic differences among applications, even though they will narrow down platform variability and provide greater interoperability.

At the level of personal healthcare systems such as the patient BAN there are serious challenges relating to security, integrity and privacy of data during transmission. These issues apply to both 
local transmission (eg. intra-BAN, BAN-PAN) and long range (e.g. extra-BAN) communications. End-to end security and Quality of Service guarantees need to be implemented. Safety of hardware (eg. electrical safety, emissions, interference) and reliability and correctness of applications must also be a priority. Comfort and convenience of sensors or BANs worn long term for continuous monitoring is important for usability and user acceptance. Timeliness of information availability in the face of unreliable performance of underlying network services is another issue. Provision of seamless services across regional and national boundaries multiplies these difficulties. Powering always on devices and continuous transmission will continue to raise technical challenges as will exploitation of the possibilities raised by ambient intelligence and ad hoc networking whilst maintaining security and privacy levels. Business models for healthcare and accounting and billing models for network services need to evolve to incorporate the new possibilities. Perhaps ironically, the bigger the potential of such services for the population at large, the bigger the problem of scalability of solutions becomes. Standardisation at all levels is essential for open solutions to prevail. Nevertheless specialization, customization and personalisation are needed, for instance for some applications BANs will have specific requirements to operate in special conditions (for example requirements to be water-proof, air tight, reusable etc.)

In the wider context, assessment and evaluation of the value of innovative $m$-health solutions and the incorporation of worthwhile $\mathrm{m}$-health technologies into existing healthcare systems in terms of structure, organization and working practices presents many future challenges. Legal issues and hospital responsibilities such as ownership of the data and legal liability are further complicated by the international dimension enabled by the opportunity of seamless access to services for free roaming patients. With new possibilities come new challenges. 